Phys. kondens. Materie 15, 346 (1973)

(C) by Springer-Verlag 1973

\title{
Erratum
}

\section{The Dielectric Constant of Liquid Metals}

\section{J. S. Helman and W. Baltensperger}

Phys. kondens. Materie 5, 60 (1966)

The formulas of the paper apply to a grand canonical ensemble; the following corrections arise from the dependence of the chemical potential on the scattering.

Eq. (16) must be replaced by

$$
\Pi^{(0)}=\frac{k^{2} N e^{2}}{m \Omega^{2}}(1-\lambda)
$$

with

$$
\lambda=\frac{4 m^{2}}{N(2 \pi)^{4}} \int_{0}^{\infty} d q q\left|V_{q}\right|^{2} S(q) \ln \left|\frac{q+2 p_{\mathrm{F}}}{q-2 p_{\mathrm{F}}}\right| .
$$

In Sect. $5, \gamma(\Omega)$ must always be replaced by $\gamma(\Omega)-\lambda$. Note that $\lambda$ cancels with the frequency independent part of $\gamma(\Omega)$. Eq. (38) must be replaced by

$$
\gamma_{0}=\frac{16 m^{2}}{3(2 \pi)^{4} N} \int_{0}^{\infty} d q q\left|V_{q}\right|^{2} S(q)\left\{\frac{1}{4} \ln \left|\frac{q+2 p_{\mathrm{F}}}{q-2 p_{\mathrm{F}}}\right|+\frac{q p_{\mathrm{F}}}{\left(2 p_{\mathrm{F}}\right)^{2}-q^{2}}\right\} .
$$

These changes do not modify the results of Appendix C. 